Correspondence

Jesús Blázquez

blazquez@cnb.csic.es

Received 29 September 2008

Revised 15 December 2008

Accepted 23 December 2008

\section{Inactivation of the $h m g A$ gene of Pseudomonas aeruginosa leads to pyomelanin hyperproduction, stress resistance and increased persistence in chronic lung infection}

\author{
Alexandro Rodríguez-Rojas, ${ }^{1}$ Ana Mena, ${ }^{2}$ Soledad Martín, ${ }^{1}$ Nuria Borrell, ${ }^{2}$ \\ Antonio Oliver ${ }^{2}$ and Jesús Blázquez ${ }^{1}$ \\ ${ }^{1}$ Centro Nacional de Biotecnología, Consejo Superior de Investigaciones Científicas (CSIC), C/ \\ Darwin 3, Campus UAM, 28049 Madrid, Spain \\ ${ }^{2}$ Servicio de Microbiologia, Hospital Son Dureta, C/ Andrea Doria 55, 07014 Palma de Mallorca, \\ Spain
}

\begin{abstract}
Clinical isolates of Pseudomonas aeruginosa that hyperproduce a dark-brown pigment are quite often found in the lungs of chronically infected patients, suggesting that they may have an adaptive advantage in chronic infections. We have screened a library of random transposon insertions in $P$. aeruginosa. Transposon insertions resulting in the hyperproduction of a darkbrown pigment were found to be located in the $h m g A$ gene, which putatively encodes the enzyme homogentisate-1,2-dioxygenase. Complementation studies indicate that $h m g A$ disruption is responsible for the hyperproduction of pyomelanin in both laboratory and clinical isolates. $\mathrm{A}$ relationship between $h m g A$ disruption and adaptation to chronic infection was explored and our results show that the inactivation of $h m g A$ produces a slight reduction of killing ability in an acute murine model of lung infection. On the other hand, it also confers decreased clearance and increased persistence in chronic lung infections. Whether pyomelanin production is the cause of the increased adaptation to chronicity or just a side effect of $h m g A$ inactivation is a question to be studied in future; however, this adaptation is consistent with the higher resistance to oxidative stress conferred in vitro by the pyomelanin pigment. Our results clearly demonstrate that $h m g A$ inactivation leads to a better adaptation to chronic infection, and strongly suggest that this mechanism may be exploited in naturally occurring $P$. aeruginosa strains.
\end{abstract}

\section{INTRODUCTION}

Pseudomonas aeruginosa is a major opportunistic pathogen that severely affects immunocompromised patients and burn victims, and causes lung infections in patients with chronic underlying diseases such as cystic fibrosis (CF), bronchiectasis and chronic obstructive pulmonary disease (COPD) (Bodey et al., 1983; Doring et al., 1987; Gilligan, 1991). Due to its extraordinary metabolic plasticity $P$. aeruginosa is capable of adapting to many different environments (Morrison \& Wenzel, 1984; Stover et al., 2000) and infecting very different types of hosts, including plants, insects and mammals (Fick, 1993; Oliver et al., 2000). Adaptation to new hosts may involve a high capacity for genetic change, thus producing new variants (Oliver et al., 2000). In this respect, the most extensively studied model is the chronic respiratory infection in CF patients, known to be driven by an intense genetic adaptation

Abbreviations: $\mathrm{CF}$, cystic fibrosis; $\mathrm{Cl}$, competition index; COPD, chronic obstructive pulmonary disease. process (Smith et al., 2006). Several mutations favour adaptation to chronic lung infection, including those leading to the hyperproduction of alginate (mucoid phenotype), reduced growth rate (small colony variant, SCV, phenotype), increased biofilm formation capacity, reduced expression of exoenzymes and increased antibiotic resistance (Driffield et al., 2008; Govan \& Deretic, 1996; Haussler et al., 2003; Oliver et al., 2000; Pritt et al., 2007; Smith et al., 2006).

Interestingly, the isolation of $P$. aeruginosa strains producing a brown pigment from CF and COPD patients is not an infrequent event: in one study about $5 \%$ of patients presented pyomelanogenic isolates (A. Oliver, unpublished results). Thus, the rationale was that these pyomelaninhyperproducer strains could have an adaptive advantage in chronic infections (the pyomelanin itself being the cause of this advantage, or simply an unrelated epiphenomenon).

Several micro-organisms have been reported to produce pyomelanins (Yabuuchi \& Ohyama, 1972), including $P$. 
aeruginosa (Nosanchuk \& Casadevall, 2003; Ernst et al., 2003). Vibrio cholerae melanin-producing mutants have been shown to be more virulent than the non-melanogenic isogenic strain (Ivins \& Holmes, 1980, 1981; Ruzafa et al., 1995), and the production of a melanin-like pigment may protect Burkholderia cepacia from host free-radicals, and thus may be considered a virulence factor (Zughaier et al., 1999).

In the current research, we first determined that the molecular basis of pyomelanin hyperproduction in $P$. aeruginosa is the inactivation of the hmgA gene. Subsequently, we verified by genetic complementation that the hyperproduction of the dark-brown pigment in two CF pyomelanogenic clinical isolates was due to the inactivation of the $h m g A$ gene. We also studied the effect of $h m g A$ disruption on adaptation to chronic infections in murine lung infection models. Finally, the possible protective role of $h m g A$ disruption and/or pyomelanin production against hydrogen peroxide and antibiotics was studied in vitro.

\section{METHODS}

Bacteria, plasmids and media. The $P$. aeruginosa strain used to construct the ISlacZ/hah insertion library was the laboratory strain PAO1 (obtained from J. L. Martínez, Centro Nacional de Biotecnología, CSIC, Madrid). Escherichia coli strain SM10pir(pIT2) was used as the transposon donor (Jacobs et al., 2003). P. aeruginosa strain PA14 and its $h m g A:: M A R 2 x T 7$ derivative were kindly provided by Dr Nicole T. Liberati (Harvard Medical School; Liberati et al., 2006). The wild-type $h m g A$ gene from PAl4 was amplified by using oligonucleotides hmgAF1 (5'-GGGCCTTGAGGATATCGG-3') and hmgAR1 (5'-AGGCGACCCAGCTACGAGTG-3') as primers of a PCR. The PCR product was cloned directly into the SmaI-digested and T-tailed pBBR1MCS-3 plasmid vector (Kovach et al., 1995). The construction was verified by sequencing and the resulting plasmid was termed pBBR1-HmgA. Plasmids pBBR1-HmgA and pBBR1MCS-3 were transformed by electroporation into E. coli S17-1 and transferred to the corresponding $P$. aeruginosa strain by conjugation, as described by Zhang et al. (1993). Growth of $P$. aeruginosa was performed in Luria-Bertani (LB) broth and on LB agar plates. Tetracycline and gentamicin were added, when necessary, to final concentrations of 150 and $15 \mu \mathrm{g} \mathrm{ml}^{-1}$ respectively. E. coli strains were routinely cultured in LB (Sambrook \& Russell, 2001) and, when appropriate, supplemented with tetracycline to a final concentration of $20 \mu \mathrm{g} \mathrm{ml}^{-1}$.

Construction and screening of the insertional library. Transposon insertions in the PAO1 chromosome were generated by following the protocol of Jacobs et al. (2003). Briefly, E. coli strain SM10pir(pIT2), containing the transposon ISlacZ/hah, was mated with $P$. aeruginosa PAO1. Mutagenized cells were selected by plating on LB agar containing tetracycline $\left(60 \mu \mathrm{g} \mathrm{ml}^{-1}\right)$ to select insertions and chloramphenicol $\left(10 \mu \mathrm{g} \mathrm{ml}^{-1}\right)$ for counterselection against the $E$. coli donor strain. After incubation for 2 days at $30{ }^{\circ} \mathrm{C}$, about 18000 tetracycline-resistant colonies were picked and inoculated into 96-well plates, each well containing $200 \mu$ freezing medium (LB broth plus $6 \%$ glycerol) supplemented with tetracycline. Plates were incubated for $48 \mathrm{~h}$ at $37{ }^{\circ} \mathrm{C}$, frozen and stored at $-80{ }^{\circ} \mathrm{C}$. Mutants producing a dark-brown pigment after incubation were isolated. Transposon insertion locations were determined by PCR and sequencing, as described by Jacobs et al. (2003).

Characterization of natural pyomelanogenic strains. Two pyomelanogenic $P$. aeruginosa clinical isolates, recovered from the sputum samples of two different patients, were obtained from the collection of the Microbiology Department of the Son Dureta Hospital. Plasmids pBBR1-HmgA and pBBR1MCS-3 were transferred from E. coli S17-1 to the corresponding strains of P. aeruginosa. PCR amplification of the $h m g A$ genes was performed using the primers described above.

Murine model of acute respiratory infection. The murine model of acute lung infection was established by following previously described protocols (Mena et al., 2007). Female C57BL/6 J mice, $20-$ $25 \mathrm{~g}$ in weight (Harlan Interfauna Ibérica), were used. Before inoculation, the mice were anaesthetized by intraperitoneal injection of ketamine (100 $\mathrm{mg} \mathrm{kg}^{-1}$; Pfizer) and xylazine $\left(10 \mathrm{mg} \mathrm{kg}^{-1}\right.$; SigmaAldrich). A vertical midline neck incision was then performed to expose the trachea of the mice, and $20 \mu \mathrm{l}$ of $2 \times$ saline-washed culture, containing approx. $1 \times 10^{6}$ exponentially growing cells of PA14, the PA14 hmgA:: MAR2xT7 mutant, or a 1:1 mixture of both strains, was transtracheally inoculated. Mouse survival was monitored daily over a 7 day period in groups of 16 mice (from two independent experiments) for each of the strains. In the competition experiments, a group of eight mice inoculated with the $1: 1$ mixture was sacrificed $48 \mathrm{~h}$ after inoculation; the lungs were aseptically extracted and homogenized in $2 \mathrm{ml} 0.9 \%$ saline solution using an IKA Ultra-Turrax T-25 disperser. Serial 10-fold dilutions were plated in duplicate on Müller-Hinton agar (MHA). PA14 and the PA14 hmgA:: MAR2xT7 mutant colonies were differentiated by their green or brown pigment respectively, and the competition index (CI) was then calculated as the mutant/wild-type ratio. In order to rule out the possibility of loss of the phenotype during in vivo growth, a representative number $(>10)$ of randomly selected colonies from each of the competition experiments were streaked on MHA with $20 \mu \mathrm{g}$ gentamicin $\mathrm{ml}^{-1}$ (MHA-Gm), to verify the presence of the resistance marker of the PA14 hmgA:: MAR2xT7 mutant. All animal experiments were approved by the Animal Ethics Committees of the University of the Balearic Islands.

Murine model of chronic lung infection. The murine model of chronic lung infection using $P$. aeruginosa-laden agarose beads was established by following previously described protocols (Macia et al., 2006; Mulcahy et al., 2008). Briefly, for the preparation of the agarose beads, $P$. aeruginosa strains were grown to late-exponential phase, washed and mixed at a 1:10 ratio with $2 \%$ agarose in PBS (pH 7.4). The mixture was added to heavy mineral oil equilibrated at $55{ }^{\circ} \mathrm{C}$, stirred for $6 \mathrm{~min}$ at room temperature and cooled for $10 \mathrm{~min}$. The resulting agarose beads were washed with 0.5 and $0.25 \%$ deoxycholic acid (sodium salt) in PBS once and then three times with PBS alone. Serial 1/10 dilutions of homogenized bead slurry aliquots were plated on MHA for a quantification of the bacterial content. Female C57BL/ $6 \mathrm{~J}$ mice, 20-25 g in weight (Harlan Ibérica), were used. The animals were specific pathogen free, and sterile water and food were provided ad libitum. Before inoculation, the mice were anaesthetized by intraperitoneal injection of ketamine $\left(100 \mathrm{mg} \mathrm{kg}^{-1}\right)$ and xylazine $\left(10 \mathrm{mg} \mathrm{kg}{ }^{-1}\right)$. A vertical midline neck incision was then made to expose each mouse's trachea, and $20 \mu \mathrm{l}$ of the bead preparation containing approximately $1 \times 10^{4}$ agarose-embedded cells was transtracheally inoculated. Mouse survival was monitored daily over a 7 day period in groups of 42 mice (from two independent experiments) for each of the strains. At 7 days post-infection animals were sacrificed. Lungs were aseptically extracted and homogenized in $2 \mathrm{ml} 0.9 \%$ saline solution using an Ultra-Turrax T-25 disperser. Serial 10-fold dilutions were plated in duplicate on MHA to calculate the c.f.u. An additional group of eight mice per strain was sacrificed $24 \mathrm{~h}$ after inoculation. Lungs were extracted, homogenized and plated, as described above. The statistical significance of the data was analysed by using Fischer's exact test, Student's $t$ test, or the MannWhitney $U$-test, as appropriate. A $P$-value of $<0.05$ was considered significant. 
Effect of $\boldsymbol{h m g A}$ inactivation and pyomelanin on peroxide resistance. Five independent exponential-phase cultures of the wild-type, the $h m g A$ variant and the complemented mutant grown in $\mathrm{LB}$ medium to $\mathrm{OD}_{600} 0.5$ were incubated at room temperature in the presence of $25 \mathrm{mM}$ or $50 \mathrm{mM}$ hydrogen peroxide $\left(\mathrm{H}_{2} \mathrm{O}_{2}\right)$ for 15,30 , 45 and $60 \mathrm{~min}$. Viable-cell counts were determined before and after treatment in both treated and untreated strains. The protective effect against peroxide of the supernatant from the pyomelanogenic strain was tested on the wild-type non-producer strain. PA14 cells obtained from $48 \mathrm{~h}$ cultures in LB were washed and resuspended in the sterilized (by filtration) supernatant from the PA14 hmgA:: MAR2xT7 pyomelanogenic mutant grown previously for $48 \mathrm{~h}$ in LB broth. As a control, identical experiments were performed using the supernatant obtained from the wild-type PA14 strain in the same conditions. The data were analysed using two-tailed unpaired $t$ tests. $P$-values of 0.05 were considered statistically significant.

Effect of pyomelanin-containing medium on antibiotic resistance. The minimal inhibitory concentrations (MICs) for ciprofloxacin, tobramycin, imipenen and ceftazidime were determined for the $h m g A$ mutant and wild-type strain PA14. Approximately $10^{3}$ cells from overnight cultures were inoculated into tubes containing $10 \mathrm{ml}$ $\mathrm{LB}$ broth and incubated at $37{ }^{\circ} \mathrm{C}$ with vigorous shaking until the midexponential phase of growth (approx. $10^{8}$ cells $\mathrm{ml}^{-1}$ ) was reached. Then $2 \times 10^{4}$ to $4 \times 10^{4}$ cells from these cultures were inoculated into each microdilution well of a microwell plate $\left(1 \times 10^{5}\right.$ to $2 \times 10^{5}$ c.f.u. $\mathrm{ml}^{-1}$ ) and incubated for $24 \mathrm{~h}$. The MIC was defined as the lowest concentration of antibiotic at which no growth could be observed. The same experiment was repeated with the wild-type strain PA14 resuspended in the pyomelanin-containing supernatant from a $48 \mathrm{~h}$ culture of the mutant $h m g A:: M A R 2 x T 7$.

\section{RESULTS AND DISCUSSION}

\section{Isolation and genetic characterization of the pyomelanin hyperproducer mutants}

Among the 18000 PAO1 mutants screened for the desired phenotype, two mutants showed a dark-brown pigment production. Transposon insertion locations were determined using the two-stage semi-degenerate PCR and sequencing described by Jacobs et al. (2003). In both cases the insertion had occurred in the same position in the $h m g A$ gene, which putatively encodes the enzyme homogentisate 1,2-dioxygenase. Since both mutants contained identical transposon insertions and showed an identical dark-brown phenotype, we took one of them for further analysis. The structure of the compounds present in the supernatant from this hyperproducer mutant (genetically uncharacterized at that time) was previously determined (Salgado \& Blazquez, 2006). These results showed that the main pigment molecule was a derivative of homogentisic acid. It is interesting to note that this structure corresponds to the dehydrogenated (oxidized) form of homogentisic acid, the main precursor of melanin in other micro-organisms such as Pseudomonas putida, Vibrio cholerae, Burkholderia cenocepacia and Shewanella colwelliana (Arias-Barrau et al., 2004; Coon et al., 1994; Kotob et al., 1995; Kovach et al., 1995). Thus, the pigment produced by the $h m g A$ mutant will be considered here as a pyomelanin.

As indicated below, for further studies we decided to use the more virulent strain PA14 and its pyomelanin hyperproducer PA14 hmgA::MAR2xT7 derivative (Liberati et al., 2006) instead of PAO1. Fig. 1(A) shows the phenotype of pyomelanin hyperproduction of PA14 hmgA:: MAR2xT7 in comparison to that of the wild-type PA14. To verify that the inactivation of the $h m g A$ gene was the only cause of pyomelanin hyperproduction, we performed a complementation study. Hyperproduction was abolished when plasmid pBBR1-HmgA, harbouring the wild-type $h m g A$ gene, was introduced into the PA14 hmgA:: MAR2xT7 mutant strain (Fig. 1B). Similarly, the introduction of plasmid pBBR1-HmgA into the hmgAdeficient strain PAO1 hmgA:: ISlacZ/hah completely abolished the production of the pyomelanin pigment (data not shown).

\section{$h m g A$, but not maiA or fahA, inactivation is the only cause of pyomelanin production}

The enzyme homogentisate 1,2-dioxygenase is involved in the pathway of phenylalanine and tyrosine catabolism. This enzyme converts homogentisic acid (2,5-dihydroxyphenylacetate) into 4-maleylacetoacetate. In Pseudomonas putida, homogentisate is catabolized via a pathway that finally yields fumarate and acetoacetate (Fig. 2A). This pathway involves three enzymes: homogentisate dioxygenase, mal-
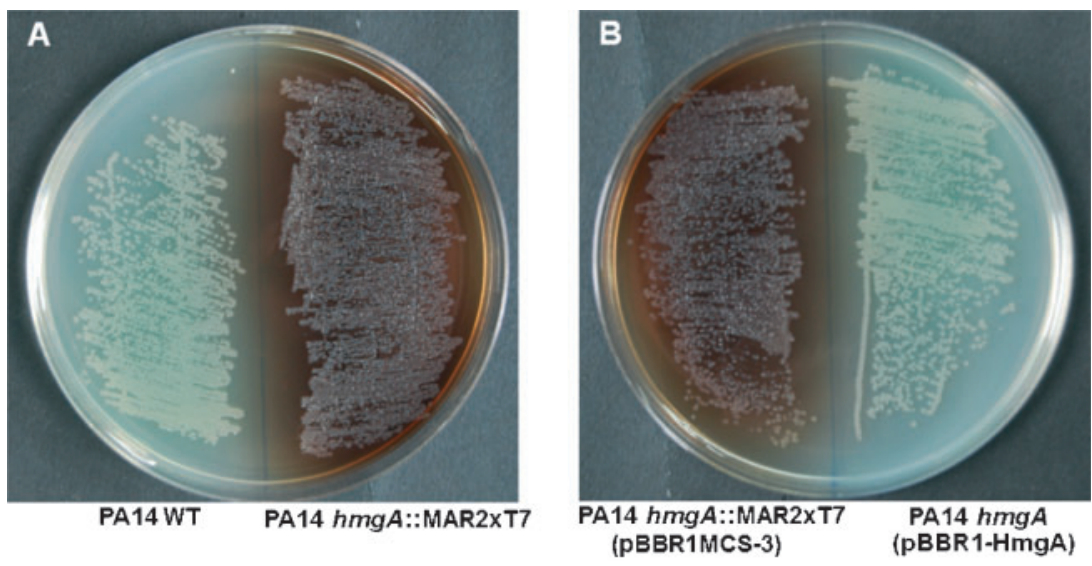

Fig. 1. (A) Phenotypic differences between PA14 and its pyomelanogenic mutant hmgA::MAR2xT7 after incubation at $37{ }^{\circ} \mathrm{C}$ for 48 h. (B) Complementation of pyomelanin hyperproduction by a plasmid harbouring the wild-type gene $h m g A$ (pBBR1-HmgA). 


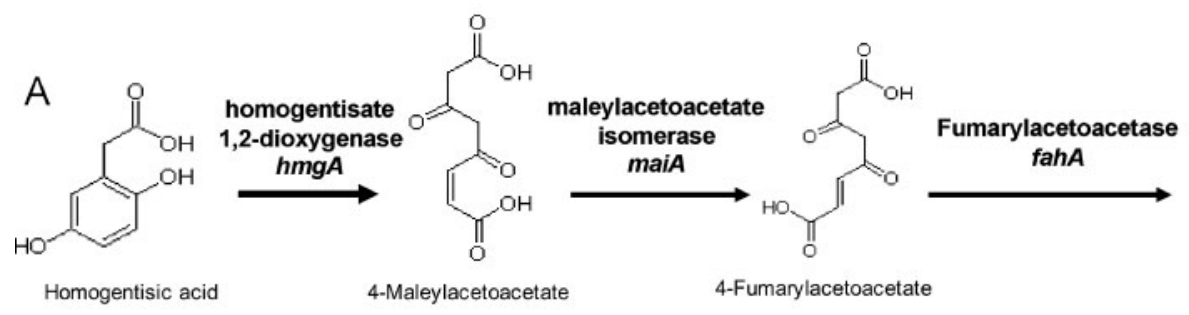

B

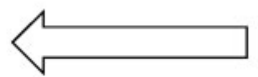

PA2006

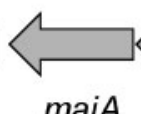

maiA

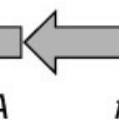

fahA

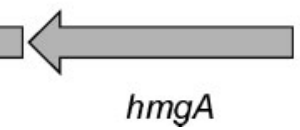

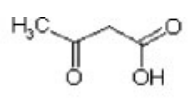

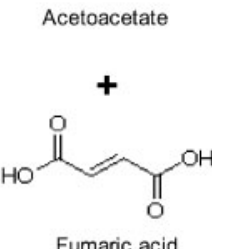

Fig. 2. Pathway for the catabolism of homogentisic acid. (A) Biochemistry of homogentisate catabolism. The enzymes and the structure of their products are indicated. (B) Arrangement of the genes putatively involved in catabolism of homogentisate in both $P$. aeruginosa PAO1 and PA14. The gene cluster encoding the homogentisate pathway is indicated by grey arrows.

eylacetoacetate isomerase and fumarylacetoacetate hydrolase. The genes encoding these enzymes appear to form a single transcriptional unit (Arias-Barrau et al., 2004). In the $P$. aeruginosa PA14 and PAO1 genomes, the genes $h m g A$, fahA and maiA (putatively encoding these three enzymes) seem to form an operon as well (Fig. 2B). In order to know if the inactivation of the other two genes of the operon, maiA and fahA, hyperproduces pyomelanin, we cultured (in both LB-agar plates and LB broth) the mutant derivatives maiA::MAR2xT7 and fahA::MAR2xT7 (Liberati et al., 2006). No visible pigment production was obtained, even after prolonged incubation for $72 \mathrm{~h}$ (data not shown). This result suggests that 4-maleylacetoacetate or any other derivative in this pathway cannot originate pyomelanin pigments in $P$. aeruginosa PA14.

Together these results demonstrate that the inactivation of the $h m g A$ gene is the only cause of the hyperproduction of the pyomelanin pigment in $P$. aeruginosa, and that neither of the other two genes ( $m a i A$ and $f a h A$ ) from the same transcriptional unit is related to pigment hyperproduction. Finally, our data suggest that, due to the lack of homogentisate 1,2-dioxygenase activity, pyomelanin formation in the $h m g A$ mutant results from the accumulation, spontaneous oxidation and polymerization of homogentisic acid.

\section{Characterization of the molecular basis of pyomelanin hyperproduction in clinical isolates of $\boldsymbol{P}$. aeruginosa}

The results presented raised the following question: is $h m g A$ inactivation the molecular basis for pyomelanin production in clinical isolates? To answer this, isolates from a series of CF patients were analysed for the production of the dark pigment. Two pyomelaninhyperproducing $P$. aeruginosa clinical isolates, recovered from the sputum samples of two CF patients, were isolated and studied. The plasmid pBBR1-HmgA, harbouring the wild-type $h m g A$ gene from the wild-type PA14 strain, was introduced into the two pyomelanogenic isolates and transconjugants were obtained in the two strains. The introduction of the wild-type $h m g A$ gene completely abolished the pigment production in these strains. PCR amplification of the $h m g A$ gene and its promoter region, using primers hmgAF1 and hmgAR1, rendered a positive result (a band of the expected size) in one of the strains only, suggesting that this strain has a minor modification in the $h m g A$ gene. The PCR-amplified $h m g A$ gene was sequenced to verify whether a mutation was present. Although some polymorphisms were found, none of them produced amino acid changes in the deduced protein, suggesting that mutations in other regions, such as transcriptional regulators, may be responsible for the observed phenotype. No PCR band was obtained in the other strain. As for the second strain, the use of $h m g A$ internal primers and PCR controls demonstrated that the $h m g A$ gene is absent in this strain and that the PCR was not inhibited in this case (data not shown). Therefore, these results strongly suggest that in both cases the lack of homogentisate 1,2-dioxygenase is responsible for the pyomelanogenic phenotype.

\section{Mortality in the murine acute lung infection model}

Pyomelanogenic strains appear to be more commonly found among clinical strains isolated from chronic infections such as CF and COPD (A. Oliver, unpublished). Thus, once it was determined that the lack of $h m g A$ activity is responsible for pyomelanin hyperproduction in both laboratory mutants and clinical isolates, we decided to study whether $h m g A$ inactivation confers an adaptive advantage in chronic infections. During the final steps of the characterization of the gene responsible for pyomelanin hyperproduction in our insertional library of PAO1, the construction of an ordered and nonredundant library of 
P. aeruginosa strain PA14 transposon insertion mutants was published by Liberati et al. (2006). We thought that since PA14 is a more virulent strain (Choi et al., 2002), it would probably be more appropriate than PAO1 for studying any differences in mortality, clearance and persistence in mouse models.

The genomic analysis of the PA14 genome had identified pathogenicity islands PAPI-1 and PAPI-2, unique to PA14 and absent in the PAO1 genome (He et al., 2004). The large set of 'extra' virulence factors encoded by these pathogenicity islands may provide some virulence and/or persistence characteristics to the PA14 strain, which are absent in PAO1. Thus, strain PA14 and its pyomelanogenic mutant hmgA:: MAR2xT7 (Liberati et al., 2006) were used to perform the mouse studies in this work.

Fig. 3 shows accumulated lung infection mortality on a daily basis for both the wild-type PA14 and PA14 hmgA: : MAR2xT7 mutant strains. Overall mouse mortality after 7 days of infection was similar for both strains, although the $h m g A$ mutant tended to kill them at a slower pace. For instance, PA14 killed $94 \%$ of the infected mice by the second day, whereas the hmgA mutant killed only $62 \%$ $(P=0.04)$. Likewise, PA14 hmgA::MAR2xT7 showed reduced fitness in the acute respiratory infection model, since it was outcompeted by the wild-type PA14 strain after 48 h of co-infection (CI $0.38, P<0.001$ ) (data not shown). This result shows that the inactivation of $h m g A$ is associated with reduced virulence in the acute infection model.

\section{hmgA disruption decreases clearance and increases persistence in the murine chronic lung infection model}

Mortality, clearance and persistence in the murine model of chronic lung infection were determined. As shown in

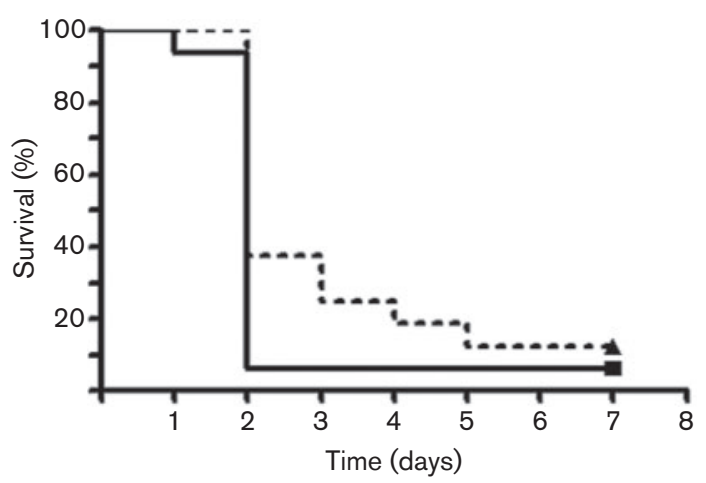

Fig. 3. Percentage survival of mice infected with PA14 $(n=16)$ or PA14 hmgA::MAR2xT7 $(n=16)$ in the mouse model of acute respiratory infection. Transtracheal inoculation of PA14 (full line, square) or PA14 hmgA::MAR2xT7 (dashed line, triangle) at $1 \times 10^{6}$ c.f.u. per mouse was performed and the percentage of surviving bacteria was monitored daily for 7 days.
Fig. 4, overall mortality, similarly to that observed in the acute lung infection model, was not significantly modified by $h m g A$ disruption in the chronic lung infection model. Moreover, in agreement with the results of the acute infection model, the $h m g A$ mutant initially showed reduced fitness in the chronic infection. The median bacterial load in the lungs after $24 \mathrm{~h}$ of infection was lower in the mutant $\left(4.8 \times 10^{3}\right.$ c.f.u. per lung $)$ than in the wildtype strain $\left(8.2 \times 10^{4}\right)$ (data not shown). However, the mutant showed decreased clearance and increased persistence after 7 days of infection (Fig. 4): $52.4 \%$ of mice infected by PA14 hmgA::MAR2xT7 showed persisting colonization after 7 days, in contrast to only $23.8 \%$ of those infected by the wild-type PA14 $(P=0.006)$. The median lung bacterial load at 7 days was similar in mice showing persisting colonization by PA14 $h m g A$ : : MAR2 $x T 7\left(2.3 \times 10^{3}\right.$ c.f.u. per lung $)$ or the wildtype PA14 strain $\left(1.3 \times 10^{3}\right)$, despite the fitness disadvantage of the $h m g A$ mutant during the first stages of the infection. Therefore, it is clear that $h m g A$ disruption conferred an adaptive advantage in chronic lung infection.

\section{Effect of $h m g A$ inactivation on resistance to $\mathrm{H}_{2} \mathrm{O}_{2}$ and antibiotics}

Protection from environmental stress has been suggested as a possible role of melanin production in micro-organisms, since the ability of free-living microbes to produce this pigment has been associated with increased survival in the environment. Melanin may protect bacteria and fungi from different types of stress, including toxic products such as $\mathrm{H}_{2} \mathrm{O}_{2}$, pesticides, heavy metals and antibiotics (Hoti \& Balaraman, 1993; Hullo et al., 2001; Nosanchuk \& Casadevall, 2006).

To test the possible role of $h m g A$ disruption on $\mathrm{H}_{2} \mathrm{O}_{2}$ resistance, we estimated the viability of cultures of strains PA14, PA14 hmgA::MAR2xT7 and the complemented mutant PA14 hmgA: : MAR2xT7(pBBR1-HmgA) subjected to $\mathrm{H}_{2} \mathrm{O}_{2}$ treatment (25 and $50 \mathrm{mM}$ ). Fig. 5(A) shows that

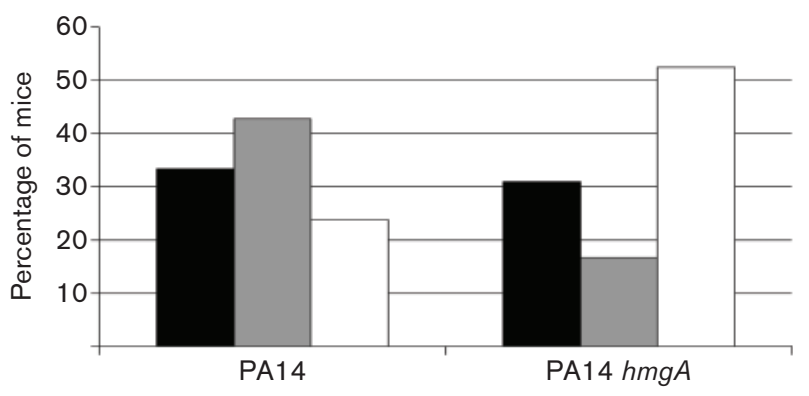

Fig. 4. Percentage of mice infected with PA14 $(n=42)$ or PA14 hmgA::MAR2xT7 $(n=42)$ that were killed (black columns), cleared of the infection (grey columns) or showed persisting colonization (white columns) 7 days after the transtracheal inoculation of $1 \times 10^{4}$ agarose-laden cells per mouse in the chronic respiratory infection model. 


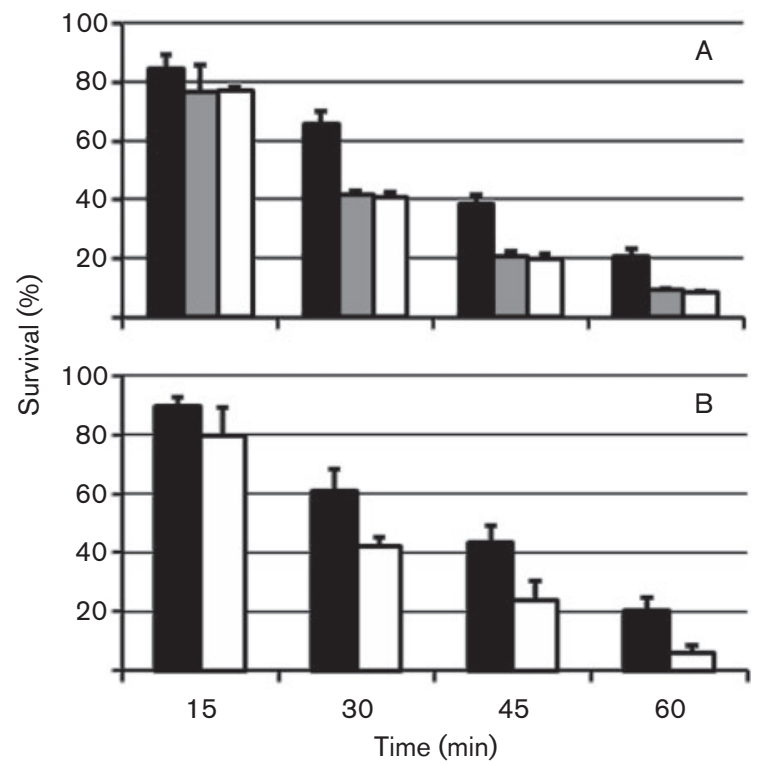

Fig. 5. Percentage of surviving bacteria after $15,30,45$ or 60 min of exposure to $50 \mathrm{mM} \mathrm{H}_{2} \mathrm{O}_{2}$. (A) Black columns, pyomelanogenic derivative PA14 hmgA: :MAR2xT7; grey columns, complemented mutant; white columns, wild-type PA14. For data obtained from wild-type versus $h m g A$ mutant, $P$-values are $0.0591,0.00012$, 0.000031 and 0.000011 after exposure for $15,30,45$ and $60 \mathrm{~min}$ respectively. For data obtained from wild-type versus the complemented hmgA mutant, $P$-values are 0.07325, 0.2721, 0.09431 and 0.3127 respectively. (B) Survival of the wild-type strain PA14 resuspended in filter-sterilized supernatant from the PA14 hmgA : MAR2xT7 pyomelanogenic mutant (black columns) or, as control, its own supernatant (white columns). Data represent the mean $\pm S D$ from five independent experiments. $P$-values for data obtained from experiments done with the supernatant from the pyomelanogenic mutant versus those done with the supernatant from the wild-type strain are 0.06732, 0.00012, 0.00003 and 0.00001 after exposure for $15,30,45$ and 60 min respectively. Differences with $P$-values $<0.05$ are considered significant.

$h m g A$ disruption protects significantly against the effect of $50 \mathrm{mM}$ peroxide after a 30,45 or $60 \mathrm{~min}$ exposure. All three strains showed similar viability after exposure to $25 \mathrm{mM}$ peroxide (data not shown).

Another possible advantage of pyomelanin hyperproduction is increased resistance to different antibiotics. This increased resistance could result in the selection of pyomelanin hyperproducer strains after prolonged treatments in chronically infected patients. To test this possibility, we measured the MICs of different currently used anti-Pseudomonas antibiotics (ceftazidime, imipenem, ciprofloxacin and tobramycin) against the wild-type and the hmgA derivative of $P$. aeruginosa PA14. No MIC differences were found among the wild-type and mutants.

In conclusion, the results of this section indicate that inactivation of the $h m g A$ gene provides protection against $\mathrm{H}_{2} \mathrm{O}_{2}$ but not antibiotics.

\section{Pyomelanin-containing supernatant is able to protect wild-type cells from $\mathrm{H}_{\mathbf{2}} \mathrm{O}_{2}$}

To gain an insight into whether the production of the pyomelanin pigment is responsible for $\mathrm{H}_{2} \mathrm{O}_{2}$ protection, wild-type non-producer PA14 cells, obtained from $48 \mathrm{~h}$ cultures in LB, were washed and resuspended in filtersterilized supernatant from the PA14 hmgA::MAR2xT7 pyomelanogenic mutant or, as control, its own supernatant (wild-type PA14), taken from cultures grown for $48 \mathrm{~h}$ in LB broth. Viable-cell counts determined before and after treatment showed that the supernatant from the mutant culture was able to protect the wild-type cells from the effect of $\mathrm{H}_{2} \mathrm{O}_{2}$ (Fig. 5B). This result suggests that the pyomelanin pigment may be the cause of such protection in $P$. aeruginosa. This interpretation is also supported by a previous report indicating that Burkholderia cepacia may derive protection against host-generated free-radicals via scavenging of these radicals by melanin (Zughaier et al., 1999). Our data may also explain why naturally occurring hyperproducer strains do not always take over the whole lung population when they appear in chronically infected patients, as non-producer strains may act as 'cheaters', and take advantage of the protection provided by hyperproducers without the associated cost (A. Oliver, unpublished).

Finally, as expected, the supernatant of the hyperproducer mutant did not provide protection against the four antibiotics tested (ceftazidime, imipenem, ciprofloxacin and tobramycin) (data not shown).

The results presented in this section show that pyomelanin hyperproduction in $P$. aeruginosa, caused by the disruption of $h m g A$, confers increased resistance to $\mathrm{H}_{2} \mathrm{O}_{2}$. The protection against reactive oxygen species (ROS) is an especially important feature for $P$. aeruginosa, as neutrophil levels are about 1500-fold higher in the infected CF lung than in uninfected individuals (Konstan et al., 1994). Macrophages and monocytes produce superoxide anions in response to engulfed bacteria or bacterial products. These ROS help to kill phagocytosed bacteria, and since the production of ROS is a fundamental mechanism of the immune system to control infection, ROS sensitivity is one of the main handicaps for bacterial proliferation (Fazal, 1997; Johansen et al., 1994). The higher in vitro resistance to oxidative stress shown in vitro by the wild-type strain when protected by the supernatant from the $h m g A$ mutant suggests that the advantage observed in vivo may be due to the hyperproduction of the pyomelanin pigment.

\section{Concluding remarks}

In conclusion, the results presented in this study demonstrate that: (i) inactivation of the $h m g A$ gene in $P$. aeruginosa leads to the hyperproduction of pyomelanin; (ii) pyomelanogenic strains isolated from CF patients seem to lack HmgA activity, as complementation with the $h m g A$ wild-type gene reverts the phenotype; (iii) an $h m g A$ mutant strain is less virulent yet more persistent than the wild-type 
in lung infection models; and (iv) hmgA mutants show increased resistance to peroxide, probably due to the protective effect of the pyomelanin pigment itself.

Despite our in vitro results with peroxide suggesting that production of pyomelanin may be the cause of increased adaptation to chronicity, whether this production is the final cause of this increased adaptation or simply a side effect of $h m g A$ inactivation is a topic to be studied in future.

The inactivation of $h m g A$ can now be included among the adaptive mutations for chronic lung infection already described in natural isolates, such as hyperproduction of alginate, reduced growth rate, increased biofilm formation capacity, reduced expression of exoenzymes and increased antibiotic resistance.

\section{ACKNOWLEDGEMENTS}

We thank Michael Jacobs, N. T. Liberati and F. M. Ausubel for kindly providing the bacterial strains. This work was supported by grants REIPI RD06/0008 (Spanish Network for Research in Infectious Diseases) and PI070215, both from the Ministerio de Sanidad y Consumo, Instituto de Salud Carlos III.

\section{REFERENCES}

Arias-Barrau, E., Olivera, E. R., Luengo, J. M., Fernandez, C., Galan, B., Garcia, J. L., Diaz, E. \& Minambres, B. (2004). The homogentisate pathway: a central catabolic pathway involved in the degradation of $\mathrm{L}$ phenylalanine, L-tyrosine, and 3-hydroxyphenylacetate in Pseudomonas putida. J Bacteriol 186, 5062-5077.

Bodey, G. P., Bolivar, R., Fainstein, V. \& Jadeja, L. (1983). Infections caused by Pseudomonas aeruginosa. Rev Infect Dis 5, 279-313.

Choi, J. Y., Sifri, C. D., Goumnerov, B. C., Rahme, L. G., Ausubel, F. M. \& Calderwood, S. B. (2002). Identification of virulence genes in a pathogenic strain of Pseudomonas aeruginosa by representational difference analysis. J Bacteriol 184, 952-961.

Coon, S. L., Kotob, S., Jarvis, B. B., Wang, S., Fuqua, W. C. \& Weiner, R. M. (1994). Homogentisic acid is the product of MelA, which mediates melanogenesis in the marine bacterium Shewanella colwelliana D. Appl Environ Microbiol 60, 3006-3010.

Doring, G., Holder, I. A. \& Botzenhardt, K. (editors) (1987). Basic Research and Clinical Aspects of Pseudomonas aeruginosa. International Symposium, Tübingen, Germany, 1986. Basel: Karger.

Driffield, K., Miller, K., Bostock, J. M., O’Neill, A. J. \& Chopra, I. (2008). Increased mutability of Pseudomonas aeruginosa in biofilms. J Antimicrob Chemother 61, 1053-1056.

Ernst, R. K., D'Argenio, D. A., Ichikawa, J. K., Bangera, M. G., Selgrade, S., Burns, J. L., Hiatt, P., McCoy, K., Brittnacher, M., Kas, A. \& other authors (2003). Genome mosaicism is conserved but not unique in Pseudomonas aeruginosa isolates from the airways of young children with cystic fibrosis. Environ Microbiol 5, 1341-1349.

Fazal, N. (1997). The role of reactive oxygen species (ROS) in the effector mechanisms of human antimycobacterial immunity. Biochem Mol Biol Int 43, 399-408.

Fick, R. B., Jr (1993). Pathogenesis and disease. In Pseudomonas aeruginosa: the Opportunist. Boca Raton, FL: CRC.

Gilligan, P. H. (1991). Microbiology of airway disease in patients with cystic fibrosis. Clin Microbiol Rev 4, 35-51.
Govan, J. R. \& Deretic, V. (1996). Microbial pathogenesis in cystic fibrosis: mucoid Pseudomonas aeruginosa and Burkholderia cepacia. Microbiol Rev 60, 539-574.

Haussler, S., Ziegler, I., Lottel, A., von Gotz, F., Rohde, M., Wehmhohner, D., Saravanamuthu, S., Tummler, B. \& Steinmetz, I. (2003). Highly adherent small-colony variants of Pseudomonas aeruginosa in cystic fibrosis lung infection. J Med Microbiol 52, 295-301.

He, J., Baldini, R. L., Deziel, E., Saucier, M., Zhang, Q., Liberati, N. T., Lee, D., Urbach, J., Goodman, H. M. \& Rahme, L. G. (2004). The broad host range pathogen Pseudomonas aeruginosa strain PA14 carries two pathogenicity islands harboring plant and animal virulence genes. Proc Natl Acad Sci U S A 101, 2530-2535.

Hoti, S. L. \& Balaraman, K. (1993). Formation of melanin pigment by a mutant of Bacillus thuringiensis H-14. J Gen Microbiol 139, 23652369.

Hullo, M. F., Moszer, I., Danchin, A. \& Martin-Verstraete, I. (2001). CotA of Bacillus subtilis is a copper-dependent laccase. J Bacteriol 183, 5426-5430.

Ivins, B. E. \& Holmes, R. K. (1980). Isolation and characterization of melanin-producing ( $m e l)$ mutants of Vibrio cholerae. Infect Immun 27, 721-729.

Ivins, B. E. \& Holmes, R. K. (1981). Factors affecting phaeomelanin production by a melanin-producing $(\mathrm{mel})$ mutant of Vibrio cholerae. Infect Immun 34, 895-899.

Jacobs, M. A., Alwood, A., Thaipisuttikul, l., Spencer, D., Haugen, E., Ernst, S., Will, O., Kaul, R., Raymond, C. \& other authors (2003). Comprehensive transposon mutant library of Pseudomonas aeruginosa. Proc Natl Acad Sci U S A 100, 14339-14344.

Johansen, H. K., Espersen, F., Cryz, S. J., Jr, Hougen, H. P., Fomsgaard, A., Rygaard, J. \& Hoiby, N. (1994). Immunization with Pseudomonas aeruginosa vaccines and adjuvant can modulate the type of inflammatory response subsequent to infection. Infect Immun 62, 3146-3155.

Konstan, M. W., Hilliard, K. A., Norvell, T. M. \& Berger, M. (1994). Bronchoalveolar lavage findings in cystic fibrosis patients with stable, clinically mild lung disease suggest ongoing infection and inflammation. Am J Respir Crit Care Med 150, 448-454.

Kotob, S. I., Coon, S. L., Quintero, E. J. \& Weiner, R. M. (1995). Homogentisic acid is the primary precursor of melanin synthesis in Vibrio cholerae, a Hyphomonas strain, and Shewanella colwelliana. Appl Environ Microbiol 61, 1620-1622.

Kovach, M. E., Elzer, P. H., Hill, D. S., Robertson, G. T., Farris, M. A., Roop, R. M., II \& Peterson, K. M. (1995). Four new derivatives of the broad-host-range cloning vector pBBR1MCS, carrying different antibiotic-resistance cassettes. Gene 166, 175-176.

Liberati, N. T., Urbach, J. M., Miyata, S., Lee, D. G., Drenkard, E., Wu, G., Villanueva, J., Wei, T. \& Ausubel, F. M. (2006). An ordered, nonredundant library of Pseudomonas aeruginosa strain PA14 transposon insertion mutants. Proc Natl Acad Sci U S A 103, 2833-2838.

Macia, M. D., Borrell, N., Segura, M., Gomez, C., Perez, J. L. \& Oliver, A. (2006). Efficacy and potential for resistance selection of antipseudomonal treatments in a mouse model of lung infection by hypermutable Pseudomonas aeruginosa. Antimicrob Agents Chemother 50, 975-983.

Mena, A., Macia, M. D., Borrell, N., Moya, B., de Francisco, T., Perez, J. L. \& Oliver, A. (2007). Inactivation of the mismatch repair system in Pseudomonas aeruginosa attenuates virulence but favors persistence of oropharyngeal colonization in cystic fibrosis mice. J Bacteriol 189, 3665-3668.

Morrison, A. J., Jr \& Wenzel, R. P. (1984). Epidemiology of infections due to Pseudomonas aeruginosa. Rev Infect Dis 6 (Suppl. 3), S627S642. 
Mulcahy, H., O'Callaghan, J., O'Grady, E. P., Maciá, M. D., Borrell, N., Gómez, C., Casey, P. G., Hill, C., Adams, C. \& other authors (2008). Pseudomonas aeruginosa RsmA plays an important role during murine infection by influencing colonization, virulence, persistence, and pulmonary inflammation. Infect Immun 76, 632638.

Nosanchuk, J. D. \& Casadevall, A. (2003). The contribution of melanin to microbial pathogenesis. Cell Microbiol 5, 203-223.

Nosanchuk, J. D. \& Casadevall, A. (2006). Impact of melanin on microbial virulence and clinical resistance to antimicrobial compounds. Antimicrob Agents Chemother 50, 3519-3528.

Oliver, A., Canton, R., Campo, P., Baquero, F. \& Blazquez, J. (2000).

High frequency of hypermutable Pseudomonas aeruginosa in cystic fibrosis lung infection. Science 288, 1251-1254.

Pritt, B., O'Brien, L. \& Winn, W. (2007). Mucoid Pseudomonas in cystic fibrosis. Am J Clin Pathol 128, 32-34.

Ruzafa, C., Sanchez-Amat, A. \& Solano, F. (1995). Characterization of the melanogenic system in Vibrio cholerae, ATCC 14035. Pigment Cell Res 8, 147-152.

Salgado, A. \& Blazquez, J. (2006). Determination of the structure of a hybrid between 2-(1,4-benzoquinone)acetic acid and a linear peptide by electrospray ionization mass spectrometry. Rapid Commun Mass Spectrom 20, 512-516.
Sambrook, J. \& Russell, D. W. (2001). Molecular Cloning: a Laboratory Manual, 3rd edn. Cold Spring Harbor, NY: Cold Spring Harbor Laboratory.

Smith, E. E., Buckley, D. G., Wu, Z., Saenphimmachak, C., Hoffman, L. R., D'Argenio, D. A., Miller, S. I., Ramsey, B. W., Speert, D. P. \& other authors (2006). Genetic adaptation by Pseudomonas aeruginosa to the airways of cystic fibrosis patients. Proc Natl Acad Sci U S A 103, 8487-8492.

Stover, C. K., Pham, X. Q., Erwin, A. L., Mizoguchi, S. D., Warrener, P., Hickey, M. J., Brinkman, F. S., Hufnagle, W. O., Kowalik, D. J. \& other authors (2000). Complete genome sequence of Pseudomonas aeruginosa PAO1, an opportunistic pathogen. Nature 406, 959-964.

Yabuuchi, E. \& Ohyama, A. (1972). Characterization of pyomelanin producing strains of Pseudomonas aeruginosa. Int J Syst Bacteriol 22, 53-64.

Zhang, C., Huang, M. \& Holloway, B. W. (1993). Mapping of the ben, ant and cat genes of Pseudomonas aeruginosa and evolutionary relationship of the ben region of $P$. aeruginosa and $P$. putida. FEMS Microbiol Lett 108, 303-309.

Zughaier, S. M., Ryley, H. C. \& Jackson, S. K. (1999). A melanin pigment purified from an epidemic strain of Burkholderia cepacia attenuates monocyte respiratory burst activity by scavenging superoxide anion. Infect Immun 67, 908-913.

Edited by: W. Bitter 